\title{
Erfahrung zählt!
}

_In den letzten Jahren konnte man dem Begriff „Evidenzbasierte Praxis“ (EBP) fast nicht ausweichen. Dem Enthusiasmus, den forschende Kollegen für dieses Konzept zeigen, stehen kritische Stimmen von Praktikern gegenüber. Womöglich haben Sie sich selbst auch schon gefragt, ob wissenschaftliche Studienergebnisse therapeutisches Können und Erfahrung ersetzen können.

_ Der Vater der EBP ist der 1934 geborene kanadische Mediziner David Sackett. Er integrierte in ihr drei Anliegen: das der Praktiker, der Forscher und vor allem der Patienten. EBP bedeutet für Sackett die Integration individueller klinischer Expertise mit der bestmöglichen externen Evidenz aus systematischer Forschung angewandt bei individuellen Patienten.

_ Mit individueller Expertise ist das Können und die Urteilskraft eines jeden Physiotherapeuten gemeint, die durch Erfahrung und praktische Tätigkeit erworben wird. Je erfahrener Physiotherapeuten sind, desto treffsicherer werden ihre therapeutischen Entscheidungen am Patienten, und desto besser können sie sich in die individuelle Situation des Patienten hineindenken.

_ Mit bester verfügbarer externer Evidenz ist klinisch relevante Forschung gemeint, insbesondere patientenorientierte Forschung mit dem Ziel, exakte Untersuchungsverfahren, aussagefähige prognostische Faktoren und wirksame rehabilitative und präventive Maßnahmen zu beschreiben.

_ Mit der individuellen Situation des Patienten ist gemeint, dass jede Therapie individuell an jeden einzelnen Patienten angepasst wird. Dieses Vorgehen ist mit dem Befolgen eines „Kochrezeptes“ zur Patientenbehandlung nicht vereinbar. Externe Evidenz könne klinische Erfahrung zwar ergänzen, aber nie ersetzen, so Sackett. Gerade die Erfahrung und Expertise eines Therapeuten entscheidet darüber, ob externe Evidenz bei einem individuellen Patienten angewandt werden kann und wenn ja, in welchem Maß die Anwendung stattfindet.

_ Nimmt man die EBP ernst, wird die Erfahrung eines Therapeuten mit der Wissenschaft Hand in Hand gehen. Ziel wird es immer sein, den Patienten, der vor einem sitzt, in diesem Moment bestmöglich zu behandeln.

Herzlichst, Ihr
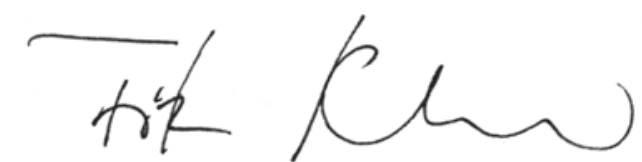